\title{
Probing atmospheric electric fields in thunderstorms through radio emission from extensive air showers
}

\author{
T.N.G. Trinh ${ }^{1 *}$, P. Schellart ${ }^{2}$, S. Buitink ${ }^{3}$, ,A. Corstanje ${ }^{2}$, J.E. Enriquez ${ }^{2}$, H. Falcke E $^{2,4,5}$, \\ J.R. Hörandel ${ }^{2,4}$, A. Nelles ${ }^{2,6}$, J.P. Rachen ${ }^{2}$, L. Rossetto ${ }^{2}$, O. Scholten ${ }^{1,7}$, S. ter \\ Veen $^{2,5}$, S. Thoudam ${ }^{2}$, A. M. van den Berg ${ }^{1}$, U. Ebert ${ }^{8,9}$, C. Rutjes ${ }^{8}$ \\ 1 KVI-CART, University Groningen, P.O. Box 72, 9700 AB Groningen, The Netherlands \\ 2 Department of Astrophysics/IMAPP, Radboud University Nijmegen, P.O. Box 9010, 6500 GL \\ Nijmegen, The Netherlands \\ 3 Astrophysical Institute, Vrije Universiteit Brussel, Pleinlaan 2, 1050 Brussels, Belgium \\ 4 NIKHEF, Science Park Amsterdam, 1098 XG Amsterdam, The Netherlands \\ 5 Netherlands Institute of Radio Astronomy (ASTRON), Postbus 2, 7990 AA Dwingeloo, The \\ Netherlands \\ 6 Now at: Department of Physics and Astronomy, University of California Irvine, Irvine, CA \\ 92697-4575, USA \\ 7 Interuniversity Institute for High-Energy, Vrije Universiteit Brussel, Pleinlaan 2, 1050 \\ Brussels, Belgium \\ 8 Center for Mathematics and Computer Science (CWI), PO Box 94079, 1090 GB Amsterdam, \\ The Netherlands \\ 9 Eindhoven University of Technology (TU/e), PO Box 513, 5600 MB Eindhoven, The \\ Netherlands \\ E-mail: t.n.g.trinh@rug.nI
}

\begin{abstract}
We present measurements of radio emission from extensive air showers taking place during thunderstorms. Their intensity and polarization patterns are different from those observed during fair-weather conditions. We introduce a simple two-layer model for atmospheric electric fields which can reproduce the main features of the intensity and polarization patterns of air shower during thunderstorms. This in turn provides a unique way to probe atmospheric electric fields.
\end{abstract}

The 34th International Cosmic Ray Conference,

30 July- 6 August, 2015

The Hague, The Netherlands

\footnotetext{
* Speaker.
} 


\section{Introduction}

Energetic cosmic rays impinging on the atmosphere create a particle avalanche called an extensive air shower. In the leading plasma of this shower electric currents are induced that generate radio waves which have been detected with LOFAR, a large array of simple antennas primarily developed for radio-astronomical observations [1]. Additionally, there is a scintillator array LORA located at the center of LOFAR to provide an independent trigger whenever an air shower with an estimated primary energy of $\geq 2 \times 10^{16} \mathrm{eV}$ is detected [2]. LOFAR has observed air showers under fair-weather conditions, so-called fair-weather showers, as well as air showers under atmospheric conditions where thunderstorms occur, so-called thunderstorm showers [3, 4].

For fair-weather showers the intensity as well as the polarization of the radio emission can be reproduced rather accurately by the standard models $[5,6,7,8,9]$. Due to the Lorentz force exerted by Earth's magnetic field $\mathbf{B}$ the relativistic electrons and positrons in the electromagnetic part of the shower are accelerated in opposite directions. These particles contribute coherently to a transverse electric current in the direction of the Lorentz force $\mathbf{v} \times \mathbf{B}$ where $\mathbf{v}$ is the propagation velocity vector of the shower. This produces a short, tens of nanosecond time scale, coherent pulse of radio emission. The emission generated by this geomagnetic mechanism is unidirectionally polarized in the $\hat{e}_{\mathbf{v} \times \mathbf{B}}$ direction $[10,5,11,12]$. A secondary emission mechanism results from a negative charge excess in the shower front. This consists of electrons knocked out from air molecules by the air shower. This also produces a short radio pulse having radial polarization with respect to the shower symmetry axis $[13,14]$. The full signals measured at the ground are the vectorial sum of the two emission components. Since the transverse-current component contributes more than the charge-excess component, the full radio emission is mainly polarized along $\hat{e}_{\mathbf{v} \times \mathbf{B}}$ with small radial diviation as shown in the left panel of Fig. 1.

For thunderstorm showers we observe large differences in the intensity and polarization patterns from the fair-weather model. We observe for these showers that it is not possible to get a good fit of the measured intensity pattern while in addition the dominant polarization direction differs from the orientation observed in the fair-weather condition [4].

We show that these differences are consequences of atmospheric electric fields. We also show that the basic effects of atmospheric electric fields on radio emission from air showers are understood and in turn measuring radio emission from thunderstorm showers provides a new tool to probe the atmospheric electric fields present in thunderclouds.

\section{Thunderstorm air showers at LOFAR}

Data for the present analysis were recorded with the low-band, 30-80 MHz, dual-polarized crossed dipole antennas located in the inner, $\sim 2 \mathrm{~km}$ radius, core of LOFAR radio telescope. Over the period between June 2011 and September 2014, LOFAR recorded a total of 762 fair-weather and thunderstorm showers [4].

Thunderstorm showers can be distinguished from fair-weather showers by three features. Firstly, the polarization patterns of the thunderstorm showers differ significantly from those of fair-weather air showers. This can be seen in the right panel of Fig. 1 where the polarization direction is clearly coherent for all antennas but no longer in the expected $\hat{e}_{\mathbf{v} \times \mathbf{B}}$ direction. There 

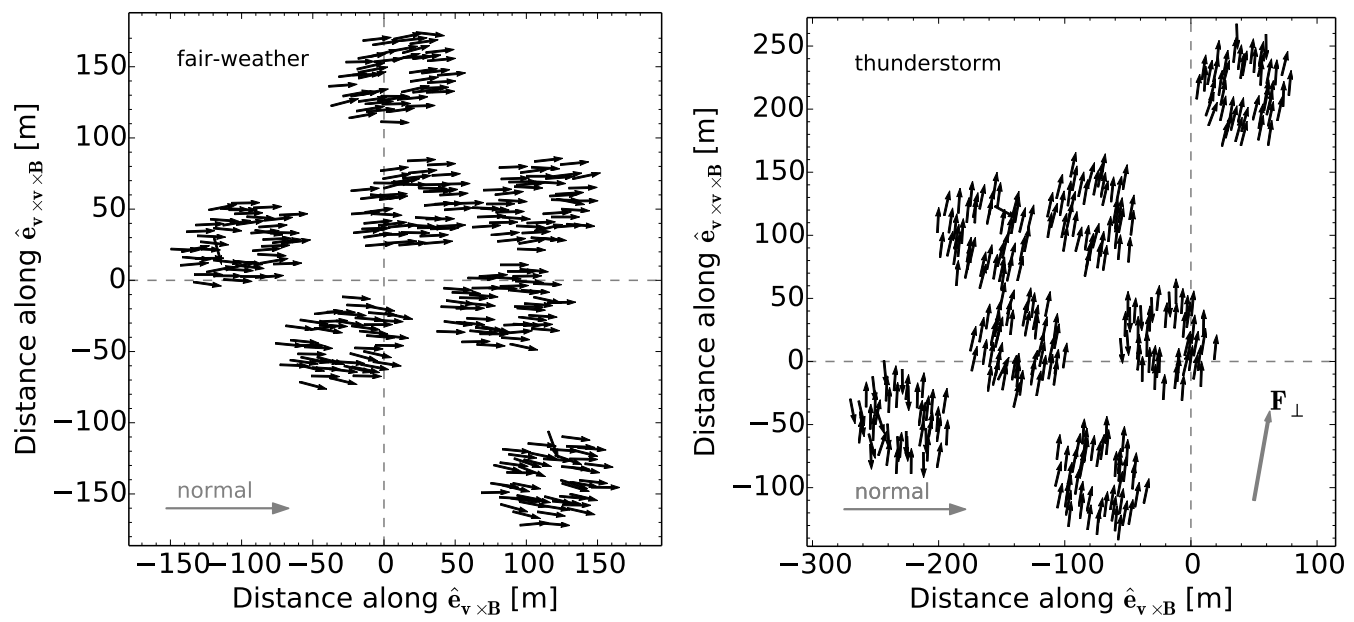

Figure 1: Polarization as measured with individual LOFAR antennas (arrows) in the shower plane for measured showers. The expected polarization direction for fair-weather air showers is indicated with "normal". The position of the shower axis, orthogonal to the shower plane, is indicated by the intersection of the dashed lines.

are 31 showers showing that their polarization differs significantly from those of fair-weather air showers. Secondly, for roughly a half of these thunderstorm showers the intensity of the radio signal at $30-80 \mathrm{MHz}$ frequencies is strongest on a ring around the shower axis with a radius of approximately $100 \mathrm{~m}$ (see Fig. 2). This "ring structure" in the intensity pattern is not present in normal fair-weather air showers which show a single maximum that is displaced in the $\hat{e}_{\mathbf{v} \times \mathbf{B}}$ direction from the shower axis due to the interference between the transverse-electric current and the charge-excess contribution $[15,16]$. The third way to distinguish the thunderstorm showers is using data from the Royal Dutch Meteorological Institute. Twenty of these 31 showers occur within $2 \mathrm{~h}$ of lightning strikes recorded within $150 \mathrm{~km}$ distance from LOFAR. Given the similarity of the polarization patterns of the remaining showers where no lightning strikes were measured, it is plausible that at these times the atmospheric electric field was not strong enough to initiate lightning. An electric field meter has since been installed at LOFAR that will provide independent verification for future measurements.

\section{A simple model of atmospheric electric fields}

Atmospheric electric fields have strong effects on the air-shower development as well as on their radio emission $[17,18]$. The atmospheric electric field along the air shower axis can be decomposed into two components

$$
\mathbf{E}=\mathbf{E}_{\|}+\mathbf{E}_{\perp},
$$

where $\mathbf{E}_{\|}$and $\mathbf{E}_{\perp}$ are parallel and perpendicular to the shower axis, respectively. The two components affect the transverse current and the charge excess in different ways. The parallel component, $\mathbf{E}_{\|}$, accelerates electrons or positrons downward, depending on its polarity, and deccelerates the other. Hence, the number of electrons and positrons increases. The additional particles, however, trail well behind the shower front, so their radio emission is not coherent in the frequency domain 

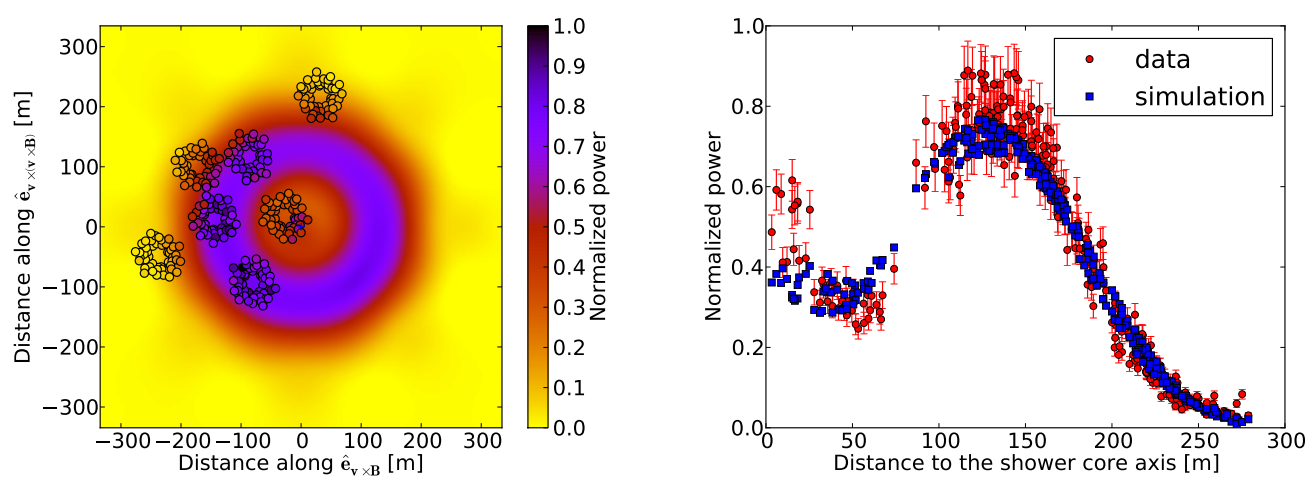

Figure 2: Radio intensity pattern during a thunderstorm. Left: the circles represent antenna positions. Their color reflects measured pulse power. The best-fitting CoREAS [8] simulation is shown in color scale in the background. Where the colors of the circles match the background a good fit is achieved. Right: measured (circles) and simulated pulse power (squares) as a function of distance to the shower axis.

of LOFAR low-band antenna (LBA). The perpendicular component, $\mathbf{E}_{\perp}$, does not affect the number of particles but changes the net force acting on the particles $\mathbf{F}=\mathbf{q}\left(\mathbf{E}_{\perp}+\mathbf{v} \times \mathbf{B}\right)$. Since the force is perpendicular to the velocity of the particle, no appreciable amount of work is done and thus the particle energy is not changed much. This force, however, changes the drift velocity of particles and thus the transverse current. The increase in the transverse velocity results in a decrease in the longitudinal velocity since the total velocity cannot exceed the speed of light. As a result, for large perpendicular electric fields, the relative velocity between the particles and the shower front becomes larger. The particles will trail further behind the shower front and their radiation is not coherent at the observed frequencies. The combination between the two effects of $\mathbf{E}_{\perp}$ results in an increase of the transverse current with the increasing applied electric fields up to $50 \mathrm{kV} / \mathrm{m}$ followed by a saturation of the current. The same trend is shown in the intensity since the intensity is proportional to the square of the current.

Based on the understanding of the influence of atmospheric electric fields on radio emission from air showers, we build a simple model of the electric fields, a two-layer model, which can reproduce the main features of the measured intensity and polarization footprints. It consists of two electric field layers where the first layer is from a higher altitude $h_{U}$ to a lower one $h_{L}$ and the second layer is between $h_{L}$ and the ground. In the upper layer, the electric field is uniform and has a unique orientation. In the second layer, the electric field polarized such that the net force changes by $180^{\circ}$ and its strength is reduced by a factor $k$. The second-layer electric field with the specific polarity introduces a destructive interference between the radio emission in the two layers which reproduces the ring structure and the unique polarization seen in the measurements.

In Fig. 2, the reconstruction of a thunderstorm event is shown. The reproduced polarization pattern is similar to the polarization pattern from the measurement shown in the right panel of Fig. 1. The reconstruction is optimal for $h_{U}=8 \mathrm{~km}, h_{L}=2.9 \mathrm{~km}$ and $k=0.55$. The reduced $\chi^{2}$ is 3.2 for the combination of radio, as measured with LOFAR, and particle data, as measured with LORA. For perfect fit of a fair-weather shower, a reduced $\chi^{2} \approx 1$ is obtained which is not reached for thunderstorm showers with a simple electric field model. However, all main features in intensity, 

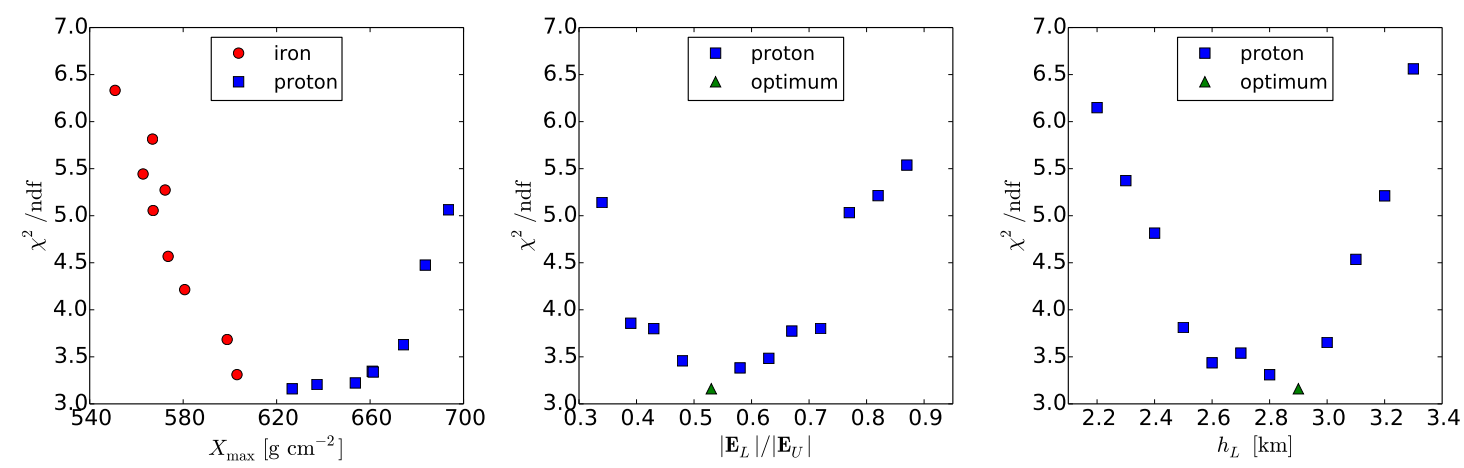

Figure 3: Sensitivity of the fit quality to variations in the atmospheric depth of shower maximum $X_{\max }$ (left panel), the relative field strength $k$ (middle panel) and the field reversal altitude $h_{L}$ (right panel). The optimal proton simulation is the same for all plots. The electric field strength, in the upper layer, for all simulations is $\left|\mathbf{E}_{\mathbf{U}}\right|=50 \mathrm{kV} / \mathrm{m}$.

polarization pattern and particle spectrum are reproduced.

The fit quality is sensitive to the parameters of the model: $h_{L}, k$ and $X_{\max }$ as shown in Fig. 3 where each parameter is varied while keeping the others fixed at the optimum values. From this the height $h_{L}$ and the relative strength of the electric fields in two layers can be derived and thus the electric field configuration can be probed. The fit quantity is sensitive to the parameter $h_{U}$ until it reaches the optimum value at $h_{U}=8 \mathrm{~km}$ and is not sensitive to higher altitudes where there are relatively few particles contributing to the radio emission since the air shower is not fully developed yet.

For fair-weather air showers the measured radio intensity is related to the simulated values through a constant scaling factor [15] giving the energy of the primary particle. This energy is derived from the particle density on the ground, as measured with LORA, combined with the information on $X_{\max }$, as determined from the radio fit. Due to the influence of the electric fields on the radio intensity, the measured intensity of thunderstorm showers is higher than the normally expected value [17]. However, the simulated intensity increases only until the atmospheric electric field strength reaches $\left|\mathbf{E}_{\mathbf{U}}\right| \sim 50 \mathrm{kV} / \mathrm{m}$ after which it starts to saturate. This saturation of the radio intensity appears to be related to the coherent nature of the emission as mentioned above.

\section{Probe atmospheric electric fields at low-frequency domain}

As mentioned in the previous section, the radio intensity in LOFAR LBA frequency domain strongly depends on the transverse electric field up to values of about $50 \mathrm{kV} / \mathrm{m}$. After this value it has little sensitivity. The effects of parallel electric fields are hardly observed in the frequency domain from $30 \mathrm{MHz}$ to $80 \mathrm{MHz}$. However, the sensitivity to atmospheric electric fields increases at lower frequencies. Fig. 4 and Fig. 5 show that at frequencies from $2 \mathrm{MHz}$ to $9 \mathrm{MHz}$, there is clear dependence of the intensity pattern on transverse electric field as well as parallel electric fields. In addition, the maximum intensity increases with increasing electric fields, so the strength of the fields can be probed more accurately in this low frequency domain. 

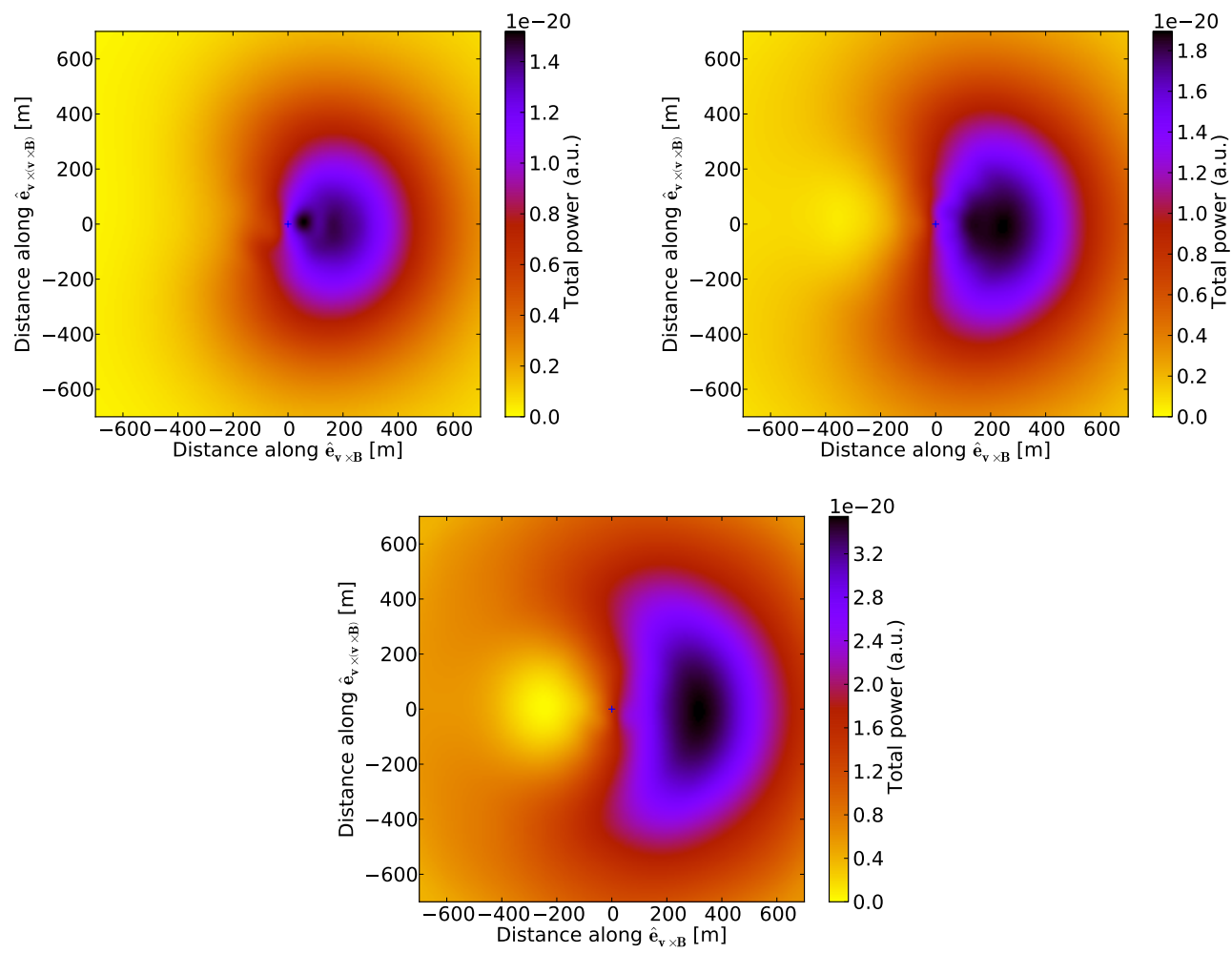

Figure 4: Intensity footprints of $10^{15} \mathrm{eV}$ vertical showers for the 2 - $9 \mathrm{MHz}$ band for the cases of no electric field (top left), $E_{\|}=50 \mathrm{kV} / \mathrm{m}$ (top right), and $E_{\|}=100 \mathrm{kV} / \mathrm{m}$ (bottom).
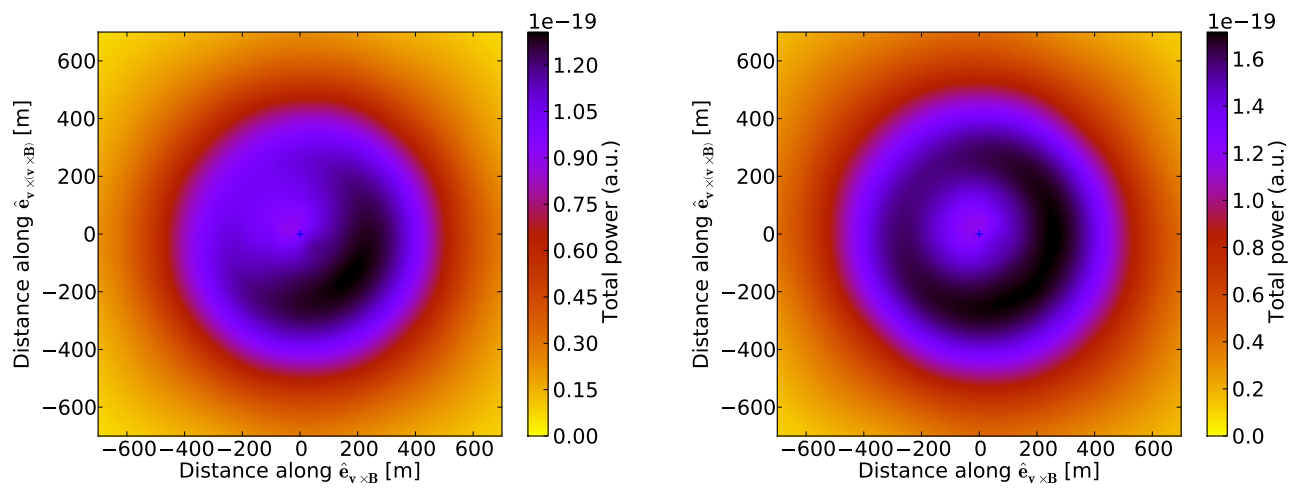

Figure 5: Intensity footprints of $10^{15} \mathrm{eV}$ vertical showers for the $2-9 \mathrm{MHz}$ band for the cases of $E_{\perp}=$ $50 \mathrm{kV} / \mathrm{m}$ (left) and $E_{\perp}=100 \mathrm{kV} / \mathrm{m}$ (right). 


\section{Summary and conclusion}

Based on a basic understanding of the effects of atmospheric electric fields on radio emission from air showers, a two-layer model of atmospheric electric fields is built and used to reconstruct thunderstorm showers. Although it is a very simple model, it can reproduce all main features observed in the intensity pattern as well as in the polarization pattern. Since the thunderstorm showers are well recontructed, they in turn help to probe the atmospheric electric fields along the shower axis present in thunderclouds. This is a unique technique because it is non intrusive and it is sensitive to a large fraction of the cloud. Since there is a large sensitivity to parallel electric fields and large transverse electric fields in the frequency range from $2 \mathrm{MHz}$ to $9 \mathrm{MHz}$, some low-frequency antennas should be installed in order to increase the sensitivity.

\section{Acknowledgements}

The LOFAR cosmic ray key science project acknowledges funding from an Advanced Grant of the European Research Council (FP/2007-2013) / ERC Grant Agreement n. 227610. The project has also received funding from the European Research Council (ERC) under the European Union's Horizon 2020 research and innovation programme (grant agreement No 640130). We furthermore acknowledge financial support from FOM, (FOM-project 12PR304) and NWO (VENI grant 639041-130). AN is supported by the DFG (research fellowship NE 2031/1-1).

LOFAR, the Low Frequency Array designed and constructed by ASTRON, has facilities in several countries, that are owned by various parties (each with their own funding sources), and that are collectively operated by the International LOFAR Telescope foundation under a joint scientific policy.

\section{References}

[1] M. P. van Haarlem et al., Lofar: The low-frequency array, A\&A 556 (2013) A2.

[2] S. Thoudam et al., Lora: A scintillator array for \{LOFAR\} to measure extensive air showers, Nuclear Instruments and Methods in Physics Research Section A: Accelerators, Spectrometers, Detectors and Associated Equipment 767 (2014), no. 0339 - 346.

[3] P. Schellart et al., Detecting cosmic rays with the lofar radio telescope, A\&A 560 (2013) A98.

[4] P. Schellart et al., Probing atmospheric electric fields in thunderstorms through radio emission from cosmic-ray-induced air showers, Phys. Rev. Lett. 114 (Apr, 2015) 165001.

[5] O. Scholten, K. Werner, and F. Rusydi, A macroscopic description of coherent geo-magnetic radiation from cosmic-ray air showers, Astroparticle Physics 29 (2008), no. 294 - 103.

[6] K. Werner, K. D. de Vries, and O. Scholten, A realistic treatment of geomagnetic cherenkov radiation from cosmic ray air showers, Astroparticle Physics 37 (2012), no. 05 - 16.

[7] J. Alvarez-MuÃśiz, W. R. C. Jr., and E. Zas, Monte carlo simulations of radio pulses in atmospheric showers using \{ZHAireS\}, Astroparticle Physics 35 (2012), no. 6325 - 341.

[8] T. Huege, M. Ludwig, and C. W. James, Simulating radio emission from air showers with coreas, AIP Conference Proceedings 1535 (2013), no. 1 128-132. 
[9] V. Marin and B. Revenu, Simulation of radio emission from cosmic ray air shower with \{SELFAS2\}, Astroparticle Physics 35 (2012), no. 11733 - 741.

[10] H. Falcke et al., Detection and imaging of atmospheric radio flashes from cosmic ray air showers, Nature 435 (2005) 313-316.

[11] K. Werner and O. Scholten, Macroscopic treatment of radio emission from cosmic ray air showers based on shower simulations, Astroparticle Physics 29 (2008), no. 6393 - 411.

[12] D. Ardouin et al., Geomagnetic origin of the radio emission from cosmic ray induced air showers observed by \{CODALEMA\}, Astroparticle Physics 31 (2009), no. 3192 - 200.

[13] Pierre Auger Collaboration, A. Aab et al., Probing the radio emission from air showers with polarization measurements, Phys. Rev. D 89 (Mar, 2014) 052002.

[14] P. Schellart et al., Polarized radio emission from extensive air showers measured with lofar, Journal of Cosmology and Astroparticle Physics 10 (2014) 14.

[15] S. Buitink et al., Method for high precision reconstruction of air shower $X_{\max }$ using two-dimensional radio intensity profiles, Phys. Rev. D 90 (Oct, 2014) 082003.

[16] A. Nelles et al., A parameterization for the radio emission of air showers as predicted by coreas simulations and applied to \{LOFAR\} measurements, Astroparticle Physics 60 (2015), no. 013 - 24.

[17] S. Buitink et al., Amplified radio emission from cosmic ray air showers in thunderstorms, A\&A $\mathbf{4 6 7}$ (2007), no. 2 385-394.

[18] W. Apel et al., Thunderstorm observations by air-shower radio antenna arrays, Advances in Space Research 48 (2011), no. 71295 - 1303. 